\title{
Fat-Soluble Vitamin
}

National Cancer Institute

\section{Source}

National Cancer Institute. Fat-Soluble Vitamin. NCI Thesaurus. Code C1550.

A vitamin that is soluble in fat solvents and oils (lipo-soluble). Fat-soluble vitamins include vitamins A, D, E, K, F. Absorbed with ingested dietary fat, fat-soluble vitamins are stored in moderate amounts from the gastrointestinal tract. Present in minute amounts in various foods, these vitamins are essential to maintaining normal metabolism and biochemical functions; fat malabsorption may result in fat-soluble vitamin deficiencies. Fat-soluble vitamins are found in many types of foods and may be supplemented with pharmaceutical formulations. ( $\mathrm{NCI04)}$ 